\title{
Dissecting Optical Response and Molecular Structure of Fluorescent Proteins With Non-canonical Chromophores
}

\author{
Breland G. Oscar ${ }^{1}$, Liangdong Zhu ${ }^{1}$, Hayati Wolfendeen ${ }^{2}$, Nikita D. Rozanov ${ }^{3}$, \\ Alvin Chang ${ }^{3}$, Kenneth T. Stout ${ }^{1,3}$, Jason W. Sandwisch ${ }^{1}$, Joseph J. Porter ${ }^{2}$, Ryan A. Mehl ${ }^{2}$ \\ and Chong Fang ${ }^{1 *}$
}

${ }^{1}$ Department of Chemistry, Oregon State University, Corvallis, OR, United States, ${ }^{2}$ Department of Biochemistry and Biophysics, Oregon State University, Corvallis, OR, United States, ${ }^{3}$ School of Chemical, Biological and Environmental Engineering, Oregon State University, Corvallis, OR, United States

Tracking the structural dynamics of fluorescent protein chromophores holds the key to unlocking the fluorescence mechanisms in real time and enabling rational design principles of these powerful and versatile bioimaging probes. By combining recent chemical biology and ultrafast spectroscopy advances, we prepared the superfolder green fluorescent protein (sfGFP) and its non-canonical amino acid (ncAA) derivatives with a single chlorine, bromine, and nitro substituent at the ortho site to the phenolate oxygen of the embedded chromophore, and characterized them using an integrated toolset of femtosecond transient absorption and tunable femtosecond stimulated Raman spectroscopy (FSRS), aided by quantum calculations of the vibrational normal modes. A dominant vibrational cooling time constant of $\sim 4$ and $11 \mathrm{ps}$ is revealed in $\mathrm{Cl}$-GFP and $\mathrm{Br}$ GFP, respectively, facilitating a $\sim 30$ and $12 \%$ increase of the fluorescent quantum yield vs. the parent sfGFP. Similar time constants were also retrieved from the transient absorption spectra, substantiating the correlated electronic and vibrational motions on the intrinsic molecular timescales. Key carbon-halogen stretching motions coupled with phenolate ring motions of the deprotonated chromophores at ca. 908 and $890 \mathrm{~cm}^{-1}$ in Cl-GFP and Br-GFP exhibit enhanced activities in the electronic excited state and blue-shift during a distinct vibrational cooling process on the ps timescale. The retrieved structural dynamics change due to targeted site-specific halogenation of the chromophore thus provides an effective means to design new GFP derivatives and enrich the bioimaging probe toolset for life and medical sciences.

Keywords: fluorescent proteins, ultrafast spectroscopy, structural dynamics, vibrational cooling, non-canonical amino acid, femtosecond stimulated Raman

\section{INTRODUCTION}

Since its discovery several decades ago, green fluorescent protein (GFP) has been widely used for biolabeling and bioimaging due to its characteristic bright green emission, high fluorescence quantum yield (FQY), and stability (Shimomura et al., 1962; Chalfie et al., 1994; Tsien, 1998; Patterson and Lippincott-Schwartz, 2002; Zimmer, 2002; Betzig et al., 2006; Fang et al., 2009; Jung, 2012b; Dedecker et al., 2013). GFP is amenable to structural alterations like circular permutation, 
leading to the development of biosensors such as the GFPcalmodulin chimera, wherein the fluorescence response is modulated by varying concentrations of free calcium ion $\left(\mathrm{Ca}^{2+}\right)$ (Baird et al., 1999; Zhao et al., 2011; Oscar et al., 2014). Due to the protein utility across broad science and engineering fields, spectroscopic studies have been performed on wild-type (wt)GFP and its derivatives to elucidate the underlying fluorescence mechanisms and predict how further structural changes may alter the optical response including unwanted events such as blinking and photobleaching. On the molecular level, excitation of neutral (A, $395 \mathrm{~nm}$ ), or anionic (B, $475 \mathrm{~nm}$ ) absorption bands of the wtGFP autocyclized Ser $_{65}{ }^{-}$ $\mathrm{Tyr}_{66}-\mathrm{Gly}_{67}$ (SYG) chromophore results in green fluorescence. After photoexcitation, the neutral chromophore $A^{*}$ undergoes a picosecond (ps) excited-state proton transfer (ESPT) reaction to reach a deprotonated intermediate state $\left(\mathrm{I}^{*}\right)$ within an unrelaxed protein environment preceding green emission (Chattoraj et al., 1996; Lossau et al., 1996; Brejc et al., 1997; Fang et al., 2009).

The optical properties of GFP can be tuned by modifying either the surrounding protein pocket in the $\beta$-barrel or the threeresidue chromophore (Table $\mathbf{S 1}$ ). For example, point mutation Thr203Tyr of the enhanced yellow fluorescent protein, EYFP, leads to red-shifted absorption and emission due to a $\pi-\pi$ interaction between spatially close tyrosine rings (Ormö et al., 1996; Wachter et al., 1998). Since the Tyr sidechain is not mechanistically required for chromophore formation, Tyr66 can be replaced: mutation to His or Trp eliminates the ESPT pathway and shifts the absorption and emission bands to generate blue and cyan fluorescent proteins, respectively (Wachter et al., 1997; Kummer et al., 2002; Ai et al., 2007; Tomosugi et al., 2009). The red fluorescent proteins are typically formed by an extended conjugation along the chromophore $\mathrm{N}$-acylimine carbonyl (Gross et al., 2000; Shaner et al., 2004; Piatkevich et al., 2010; Subach and Verkhusha, 2012). Because these strategies in tuning GFP spectral properties conventionally involve only 20 standard amino acids, they pose certain limitations in achieving desired properties. Notably, the site-specific modification of proteins with non-canonical amino acids (ncAAs) provides an appealing way to engineer spectral properties and encode new functionalities (Link et al., 2003; Wang et al., 2003; Peeler and Mehl, 2012). The GFP chromophore with a $p$-azido-Lphenylalanine mutation, for example, exhibits photoactivatable behavior originating from phenyl azide photolysis in the unnatural chromophore (Reddington et al., 2013). The ncAAs can further act as site-specific vibrational probes or spin labels, making them ideal for structural dynamics techniques such as electron paramagnetic resonance (EPR) spectroscopy, NMR, and time-resolved vibrational spectroscopy (Fleissner et al., 2009; Sripakdeevong et al., 2014; Hall et al., 2019).

In this work, we characterized a series of superfolder GFP (sfGFP) (Pédelacq et al., 2006) mutants that contain a single ncAA point mutation at the chromophore tyrosine residue and compared their attributes to model chromophores using both ultrafast electronic and vibrational spectroscopic signatures (Fang et al., 2018). The halogenated derivatives of sfGFP, 3chlorotyrosine (Cl-GFP) and 3-bromotyrosine (Br-GFP), contain an electron-withdrawing substituent at the ortho site to the phenolic hydroxyl which introduces steric effects in the protein pocket and increases the polarizability of the aromatic bonds over the chromophore ring system. Meanwhile, the 3-nitrotyrosine (nitro-GFP) mutant contains a strong electron-withdrawing group capable of forming additional hydrogen bonds in addition to an $\sim 30 \AA^{3}$ increase in residue volume (De Filippis et al., 2006). Spectral properties such as absorption, emission, and excitedstate dynamics are characterized by steady-state and timeresolved electronic spectroscopy; in addition, the chromophore structure and local environment can be revealed by femtosecond stimulated Raman spectroscopy (FSRS) (Dietze and Mathies, 2016; Fang et al., 2019). This integrated experimental platform resolving the coupled electronic and atomic motions in highly fluorescent systems allows us to better understand the effect of a ncAA mutation at the active site, which elucidates the conformational preference of a chromophore inside the protein matrix and the underlying photophysics/photochemistry of fluorescent proteins in the electronic excited states.

\section{MATERIALS AND METHODS}

\section{Protein Preparation}

The incorporation of a nitrotyrosine (Cooley et al., 2014; Rauch et al., 2016) or halotyrosine (Jang et al., 2020) at a selected position of sfGFP was performed as previously described. Briefly, the codon codifying each of the tyrosine residues in the sfGFP sequence optimized for bacterial expression was replaced by an amber stop codon (TAG), which was recognized by the orthogonal nitro or halotyrosine-bearing suppressor tRNA and engineered tRNA synthetase. The modified proteins were then expressed, purified, and confirmed by mass spectrometry (Jang et al., 2020). The protein concentrations for our ultrafast spectroscopic characterization were $10 \mathrm{mg} / \mathrm{mL}$ at $\mathrm{pH}=8.1$ (10 mM Tris, $\left.50 \mathrm{mM} \mathrm{Na}_{2} \mathrm{HPO}_{4}, 100 \mathrm{mM} \mathrm{NaCl}\right)$ and $5.5(100 \mathrm{mM}$ citric acid, $200 \mathrm{mM} \mathrm{Na} \mathrm{HPO}_{4}, 100 \mathrm{mM} \mathrm{NaCl}$ ). As control samples, the monohalogenated 4-hydroxybenzylidene-1,2dimethylimidazolinone (HBDI) chromophores were synthesized according to literature by combining an iminoglycine methyl ester with a Schiff base (Baldridge et al., 2010), as detailed in the Supplementary Text.

\section{Spectroscopic Methods}

The UV/Visible and emission spectra of all proteins and small molecules were collected on a ThermoScientific Evolution 201 and Hitachi F-2500 fluorescence spectrophotometer, respectively. Quantum yield was measured relative to fluorescein in $0.1 \mathrm{M}$ $\mathrm{NaOH}$ according to the reported method (Patterson et al., 1997). The tunable picosecond (ps) Raman pump, white light probe, and femtosecond (fs) actinic pump (see Supplementary Text) (Zhu et al., 2014; Liu et al., 2016) enable the acquisition of time-resolved FSRS data in the electronic excited state with simultaneously high spectral and time resolutions (Dietze and Mathies, 2016; Fang et al., 2018). Transient absorption (TA) spectra were collected before each experiment with the Raman pump blocked so that only the fs pulses interact with the sample. The UV/Visible spectra were recorded before and after timeresolved experiments to check sample integrity $(<5 \%$ change 


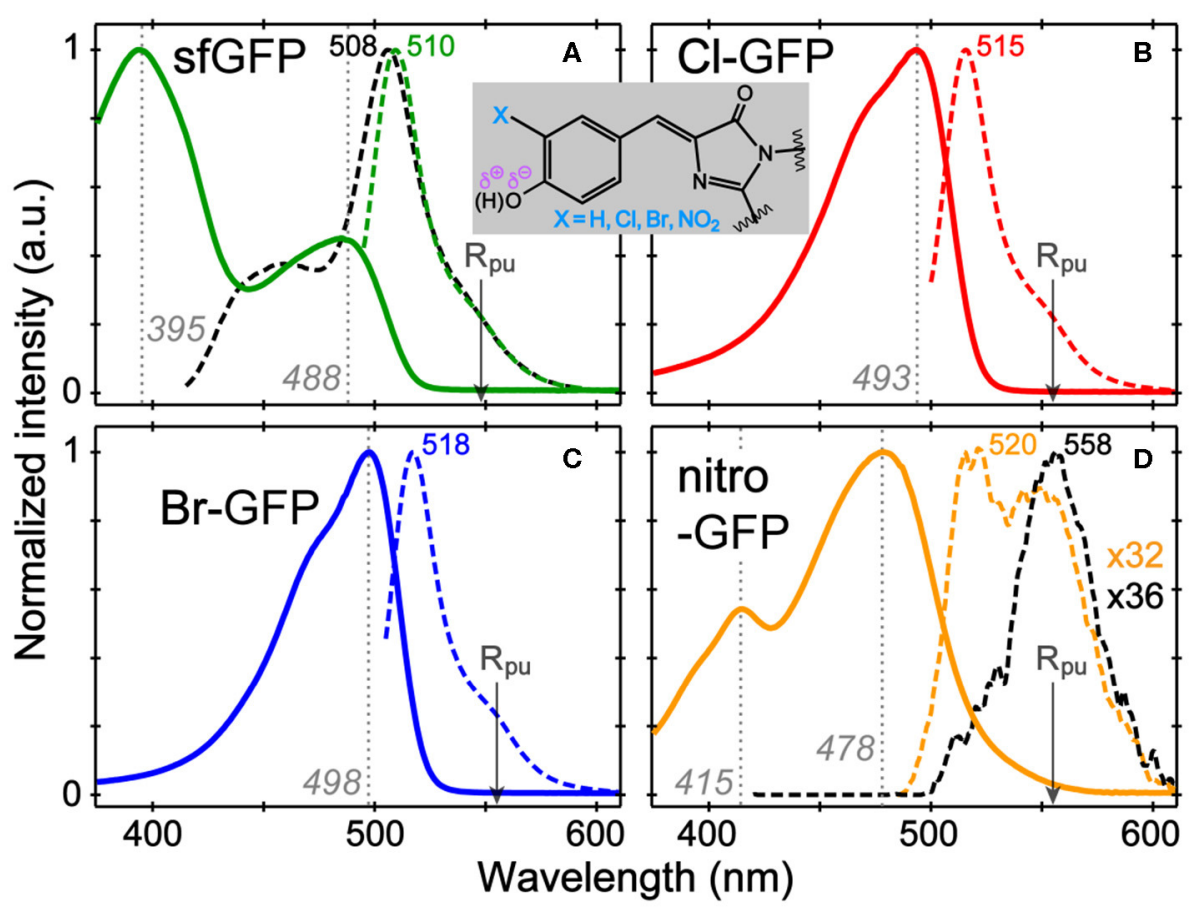

FIGURE 1 | Normalized absorption (solid) and emission (dashed lines) spectra of (A) sfGFP, (B) Cl-GFP, (C) Br-GFP, and (D) nitro-GFP in pH = 5.5 aqueous buffer solution. The excitation wavelengths are marked by gray dotted lines. For sfGFP and nitro-GFP, emission after the bluer excitation is shown as black dashed lines. The nitro-GFP emission normalization factor is denoted to manifest weak emission. The Raman pump is indicated by the arrow. The chromophore chemical structure is shown in the inset.

commonly detected). A full description of the methods can be found in our earlier reports on fluorescent proteins (Tang et al., 2016, 2018a; Fang et al., 2018).

\section{RESULTS AND DISCUSSION}

\section{Steady-State Electronic Spectroscopy}

The absorption and emission spectra of sfGFP, Cl-GFP, BrGFP, and nitro-GFP in $\mathrm{pH}=5.5$ buffer solution (Figure 1) are tabulated in Table S2. The $\mathrm{p} K_{\mathrm{a}}$ of sfGFP is $\sim 6$ so a distinct neutral chromophore population $\left(\lambda_{\max }=395 \mathrm{~nm}\right)$ is observed (Figure 1A), whereas in more alkaline conditions the deprotonated chromophore population $\left(\lambda_{\max }=488 \mathrm{~nm}\right)$ dominates (Figure S1A). We observed a lowered $\mathrm{p} K_{\mathrm{a}}$ of $\sim 4.5$ upon tyrosine halogenation inside sfGFP, so the neutral chromophore absorption around $400 \mathrm{~nm}$ is largely absent at $\mathrm{pH}=5.5$ while the anionic chromophore absorption bands red-shift (Figures S1B,C). These results are corroborated by the $\mathrm{p} K_{\mathrm{a}}$ of $3 \mathrm{Cl}$-Tyr EGFP at $\sim 4.7$ and red-shifted spectral peaks vs. EGFP (Ayyadurai et al., 2012; Zhang et al., 2012). The emission profiles of Cl-GFP and Br-GFP are also redshifted vs. sfGFP, and the shift magnitude increases with the mass of the halogen substituent (Figures 1A-C), the trend matching 3-iodotyrosine-GFP with a red-shifted emission beyond $520 \mathrm{~nm}$ (Young et al., 2011). Notably, changes in absorption and emission for chromophores in a protein matrix are more pronounced than those in small molecules. The absorption bands of the sfGFP model chromophore, neutral, and anionic $p$-HBDI with capped methyl groups, are found at 370 and $425 \mathrm{~nm}$, respectively (Vengris et al., 2004; Taylor et al., 2019). The corresponding $\mathrm{Cl}$ (Br)-HBDI bands appear at $369(368) \mathrm{nm}$ and $424(425) \mathrm{nm}$ in $\mathrm{pH}=3$ and 7.6 aqueous solutions, respectively.

In contrast, the nitrated GFP chromophore results in a non-fluorescent protein, which is not surprising given the photochemistry of nitrated aromatics (see Supplementary Text for the mechanism; De Filippis et al., 2006; Tang and Fang, 2019). The absorption spectrum shows two bands at 415 and $478 \mathrm{~nm}$ that exhibit a $\mathrm{pH}$-dependent ratio change with the $415 \mathrm{~nm}$ peak becoming stronger under acidic conditions (Figure S1D). The $415 \mathrm{~nm}$ band likely corresponds to neutral chromophore in nitro-GFP and is red-shifted from the $395 \mathrm{~nm}$ absorption band in sfGFP, which could be due to an intramolecularly $\mathrm{H}$-bonded form in nitro-GFP. Excitation of either absorption band produces negligible fluorescence $(\Phi<0.0005$, see Table S2) and the detectable emission is significantly red-shifted to $\sim 550 \mathrm{~nm}$. This behavior is reminiscent of free 3-nitrotyrosine in solution, which shows the $\mathrm{pH}$-dependent changes of its visible absorption bands, a $\mathrm{p} K_{\mathrm{a}}$ near neutral $\mathrm{pH}$, and an FQY below 0.0001 (Tang and Fang, 2019), leading to the effective use of 3-nitrotyrosine as a FRET quencher in peptides and proteins when a nearby fluorescent residue (e.g., Tyr, Trp) acts as the donor (Duus et al., 1998; De Filippis et al., 2006). 


\section{Stimulated Raman Spectroscopy in the Electronic Ground and Excited States}

To verify the chromophore's ionization state and uncover local interactions within the protein pocket, we implemented the wavelength-tunable FSRS technique at different resonance conditions (Liu et al., 2016; Fang et al., 2018), wherein a narrowband Raman pump and broadband Raman probe induce the stimulated Raman scattering signal with desirable enhancement to achieve high signal-to-noise ratio. The ncAA chromophores exhibit unique spectral signatures when compared to sfGFP; for example, the halogenated chromophores contain highly polarizable groups that affect Raman peak frequencies, and the nitrated chromophore consists of the spectrally isolated $-\mathrm{NO}_{2}$ vibrational modes that act as sensitive probes for the local environment (De Filippis et al., 2006; Ayyadurai et al., 2012).

In the ground-state FSRS of the protein series in $\mathrm{pH}=$ 5.5 buffer (Figure 2), the Raman pump is energetically close to the absorption band of the anionic chromophore (Figure 1). In sfGFP, most of the Thr-Tyr-Gly (TYG) chromophore population is neutral, but the pre-resonance condition favors the anionic subpopulation (see Figure S1A) and amplifies its Raman features. The protonation state is confirmed by the 1,547 $\mathrm{cm}^{-1}$ marker band, attributed to the $\mathrm{C}=\mathrm{N}, \mathrm{C}=\mathrm{C}$, and $\mathrm{C}=\mathrm{O}$ stretching motions in the anionic chromophore (see Table S3 for vibrational normal mode assignments) based on literature and our calculations (Bell et al., 2000; Schellenberg et al., 2001; Tozzini and Nifosì, 2001). For proteins with primarily neutral chromophores, this marker band shifts to $\sim 1,566 \mathrm{~cm}^{-1}$, also observed in wtGFP (Fang et al., 2009), a series of GFP-based $\mathrm{Ca}^{2+}$ biosensors (Oscar et al., 2014; Tang et al., 2016), and in sfGFP at an off-resonance condition (Figure S2A). The ncAAmutant proteins (Figures $2 \mathrm{~A}-\mathbf{C}$, also see Figure S2B for the off-resonance FSRS data of Br-GFP) all exhibit strong peaks near $1,542 \mathrm{~cm}^{-1}$, corroborating the anionic chromophore as determined by the electronic absorption spectra (Figure 1). The $\sim 1,576 \mathrm{~cm}^{-1}$ shoulder peak in mutant proteins is assigned to additional phenolate ring $\mathrm{C}=\mathrm{O}$ and $\mathrm{C}=\mathrm{C}$ stretch contributions in the anionic chromophore (Tables S4, S5), but this mode may indicate an $\mathrm{H}$-bonded population of halogenated chromophores while the $\mathrm{H}$-bond partner could be an adjacent water or protein residue in forming the $\mathrm{O}-\mathrm{H} \cdots \mathrm{X}(\mathrm{X}=\mathrm{Cl}, \mathrm{Br})$ bond (Pal et al., 2005). In the anionic chromophores outside the protein matrix, strong $\mathrm{C}=\mathrm{C}$ and $\mathrm{C}=\mathrm{O}$ stretching modes appear at $1,560 \mathrm{~cm}^{-1}$ (Cl-HBDI) and $1,558 \mathrm{~cm}^{-1}$ (Br-HBDI) in Figure S3, slightly blue-shifted from the reported $1,556 \mathrm{~cm}^{-1}$ mode of HBDI in basic solution (Bell et al., 2000; Schellenberg et al., 2001; Taylor et al., 2019).

The cluster of modes between $\sim 1,200$ and $1,400 \mathrm{~cm}^{-1}$ also probe the chromophore's protonation state: the $1,256 \mathrm{~cm}^{-1}$ mode in sfGFP involves the phenolate ring $\mathrm{H}$-rock and $\mathrm{CO}$ stretch, which typically exhibits a frequency blueshift in the deprotonated state (Bell et al., 2000; Fang et al., 2009; Oscar et al., 2014). This mode blue-shifts to $1,261 \mathrm{~cm}^{-1}$ in ClGFP and Br-GFP, consistent with the incorporation of an electron-withdrawing group adjacent to the phenolate oxygen site and the increased acidity as well as photoacidity of the chromophore (Chen et al., 2019). In contrast, for the phenolate ring $\mathrm{H}$-rocking and imidazolinone ring $\mathrm{C}-\mathrm{N}$ stretching mode at $1,369 \mathrm{~cm}^{-1}$ (Table S3) that was also observed for the TYG chromophore inside a protein $\mathrm{Ca}^{2+}$-biosensor (Tang et al., 2016), due to steric hindrance this mode red-shifts to $\sim 1,360$ $\mathrm{cm}^{-1}$ upon chromophore halogenation (Figure 2). Moreover, the $1,167 \mathrm{~cm}^{-1}$ phenolate ring $\mathrm{H}$-scissoring motion exhibits a notable blueshift to $\sim 1,192 \mathrm{~cm}^{-1}$ in Cl-GFP and Br-GFP since the pertinent normal modes of the halogenated chromophores involve less imidazolinone ring contributions (Tables S3-S5). These in-plane vibrational motions thus serve as sensitive probes to elucidate the effect of ortho-halogenation of the largely planar chromophore inside a protein pocket.

Notably, the chromophore autocyclization during protein maturation is primarily a function of the protein backbone. A majority of GFP chromophores are observed in the $Z$ (cis) stereoisomer, but the halogen substituent on tyrosine can occupy two distinct atropisomeric positions with the probability of each determined by the properties of the substituent itself as well as the local environment supplied by the protein interior (Bae et al., 2004; Pal et al., 2005; Jung, 2012a; Chang et al., 2019). For example, the crystal structure and electron density mapping of 3-fluorotyrosyl-EGFP revealed two conformations of the TYG chromophore with a major conformer wherein fluorine interacts with Thr203, equivalent to Configuration 1 in Figure 3 (Bae et al., 2004). Small-molecule analogs of the chromophore were reported with this conformation as well as the Trp-containing chromophores and the 3,4-dihydroxyL-phenylalanine GFP chromophore (Hyun Bae et al., 2003; Hasegawa et al., 2007; Ayyadurai et al., 2011). However, 3chlorotyrosine chromophores in the short H-bond (His148Asp) GFP system exhibit only one crystallographic occupancy corresponding to Configuration 2 in Figure 3 partly due to specific electrostatic interactions introduced by the nearby His148Asp mutation (Oltrogge and Boxer, 2015). Can FSRS provide evidence for the protein chromophore configuration?

Several low-frequency motions below $1,000 \mathrm{~cm}^{-1}$ are resolved for sfGFP and the mutated series (Figure 2), and these modes are sensitive to the chromophore conformation. The $\mathrm{C}-\mathrm{Cl}$ stretch (550-800 $\left.\mathrm{cm}^{-1}\right), \mathrm{C}-\mathrm{Br}$ stretch $\left(500-700 \mathrm{~cm}^{-1}\right)$, and $\mathrm{NO}_{2}$ bend $\left(\sim 820 \mathrm{~cm}^{-1}\right)$ are all expected to occur in this region (Kovács et al., 1998; Chen et al., 2020). Mode assignment of the anionic TYG chromophore and the halogenated derivatives in both conformations (Figure 3) was carried out by comparing the experimental Raman spectra to literature values and density functional theory (DFT) RB3LYP-level calculations with the 6$311 \mathrm{G}+(\mathrm{d}, \mathrm{p})$ basis set performed in vacuo and in water. Strong vibrational modes at $908 \mathrm{~cm}^{-1}$ in Cl-GFP and $890 \mathrm{~cm}^{-1}$ in $\mathrm{Br}-$ GFP are assigned to phenolate ring breathing with a prominent $\mathrm{C}-\mathrm{X}$ stretching component. The ring breathing mode has been previously assigned at $\sim 820 \mathrm{~cm}^{-1}$ (Oscar et al., 2014) that is also listed in Table S3, but the C-X stretching contribution blueshifts the calculated normal mode frequency (see Tables S4, S5). Similarly, the benzene ring breathing mode at $992 \mathrm{~cm}^{-1}$ was experimentally recorded at $1,001 \mathrm{~cm}^{-1}$ in chlorobenzene and 998 


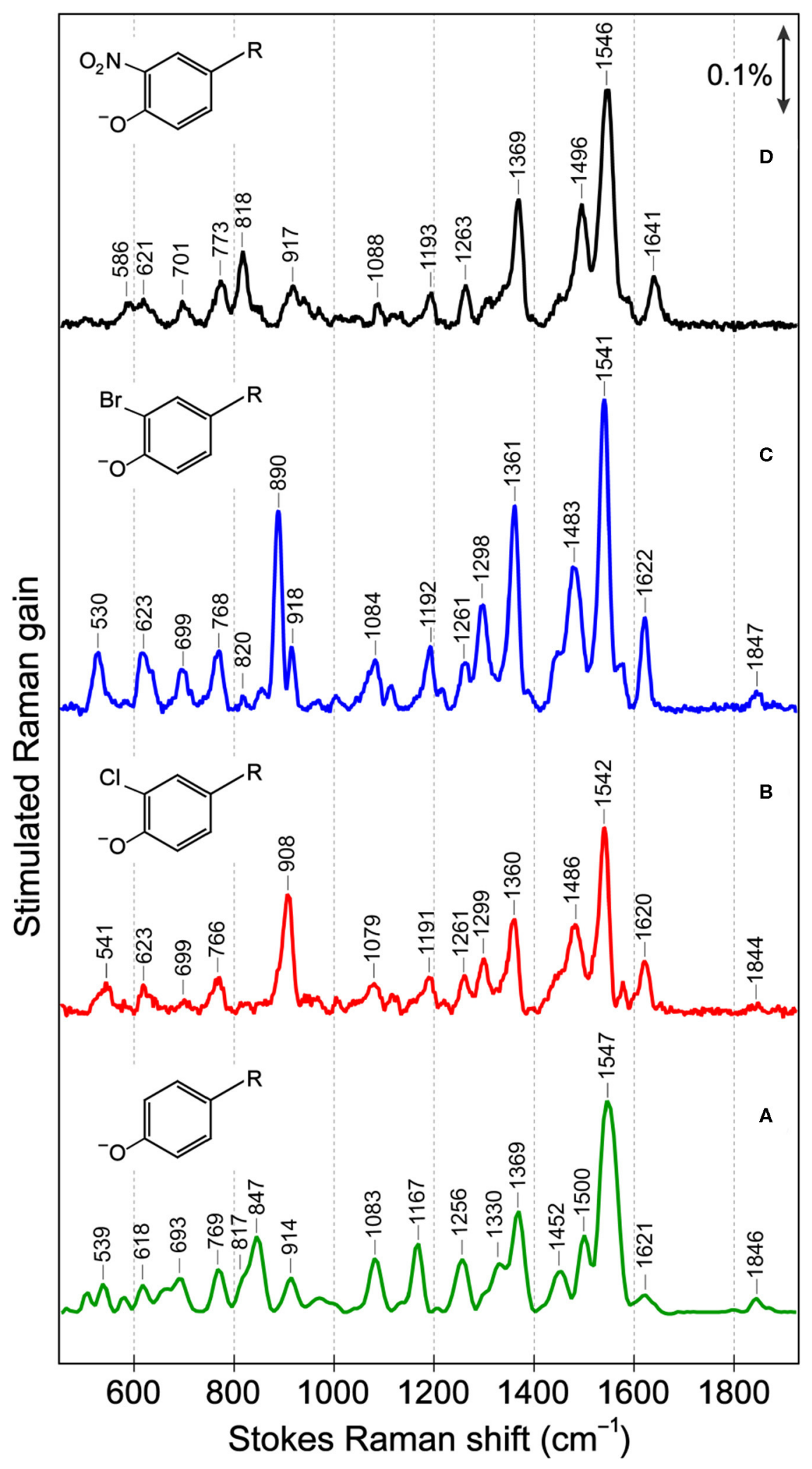

FIGURE 2 | Ground-state Stokes FSRS data of (A) sfGFP, (B) Cl-GFP, (C) Br-GFP, and (D) nitro-GFP in pH = 5.5 aqueous buffer solution. In (B-D) the Raman pump was set at $555 \mathrm{~nm}$ while in (A) the Raman pump wavelength was $548 \mathrm{~nm}$. The stimulated Raman gain is indicated by the double-headed arrow. The anionic chromophore chemical structures with various substitutions are depicted in the inset. $R$ represents the remaining conjugated framework of the chromophore that connects to protein backbone. 


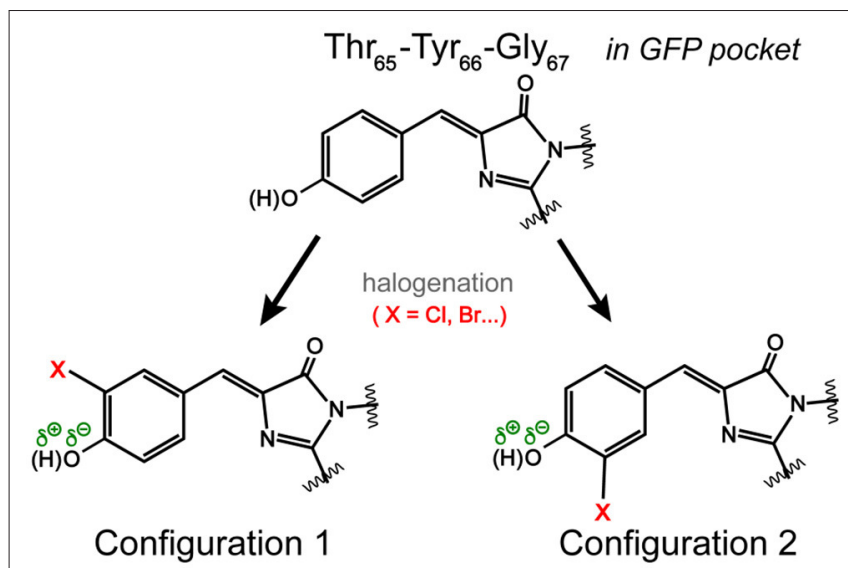

FIGURE 3 | Possible orientations of the halogen atom $(X)$ on the GFP chromophore. In Configuration 1, the halogen atom is oriented toward Thr203 in a typical GFP protein matrix. In Configuration 2, the halogen atom could face Ser205 (Pal et al., 2005). The dissociable hydroxyl group is highlighted.

$\mathrm{cm}^{-1}$ in bromobenzene, so this mode is a useful reporter on halogen substitution (Meneely et al., 1971).

Resonance Raman spectra with a Raman pump close to the red edge of electronic absorption bands (Figure 1, Figure S1) were used to further assign the low-frequency vibrational bands (Figures 4A,B, Figure S4), supported by the anti-Stokes FSRS with a $580 \mathrm{~nm}$ Raman pump (Figure S5) (Tang et al., 2018b). With identical protein concentration and Raman pump power, the signal strength is increased by an order of magnitude with $507 \mathrm{~nm}$ pump. Resonance Raman peaks include excitedstate contributions (Quick et al., 2015), which can be seen by strong agreement between the resonance Raman spectra and the excited-state spectra at $50 \mathrm{fs}$ following $480 \mathrm{~nm}$ photoexcitation. Since halogen atoms are expected to increase chromophore polarizability, the modes strongly influenced by halogenation are enhanced (see Supplementary Text). The lineshape of highfrequency modes is largely preserved in all proteins, though the peaks in $S_{1}$ are generally broader than those in $S_{0}$ due to the shorter lifetime of the excited-state species, while the frequency shift is due to an interplay between anharmonicity and the intrinsic frequency change from $S_{0} \rightarrow S_{1}$ due to electron redistribution (Chen et al., 2018; Fang et al., 2018).

Based on the ground-state FSRS and calculations, we tentatively assign Br-GFP to Configuration 1 in Figure 3 such that bromine interacts with the nearby Thr203 residue. Though DFT calculations of the model chromophore in vacuo cannot capture the myriad of interactions between the protein pocket and the chromophore, there is better agreement between the experimental and calculated vibrational modes of Configuration 1 of Br-GFP, especially in the low-frequency region (Merrick et al., 2007; Wang et al., 2015). In particular, the observed 890 and $918 \mathrm{~cm}^{-1}$ modes (Figure 2C) both have significant $\mathrm{Br}$ contributions and the calculated frequencies of 852 and $881 \mathrm{~cm}^{-1}$ in Configuration 1 (Table S5) better match the experimental energy gap of $28 \mathrm{~cm}^{-1}$ between the modes, instead of the exact mode frequencies that are highly subjective to the frequency scaling factor (Merrick et al., 2007). A crystallographic analysis is required to confirm if a minor population exists; however, we expect the bromine to experience more repulsive interactions with the sidechains of Ser205 and Glu222 in Configuration 2 based on the sfGFP crystal structure (PDB ID: 2B3P) (Pédelacq et al., 2006). Our preliminary molecular dynamics simulations based on free energy perturbation methods (Seeliger and de Groot, 2010) with a calculated chromophore force field (Malde et al., 2011), however, seem to suggest that while both configurations are stable in the protein pocket, Configuration 2 of Cl-GFP is thermodynamically more favorable. Further investigation is thus needed to better determine which configuration is dominant surrounded by dynamic protein residues in the system of interest.

\section{Excited-State Electronic and Structural Dynamics of the Halogenated sfGFP}

While the small molecule analog HBDI undergoes a nonradiative cis-to-trans isomerization after photoexcitation (Mandal et al., 2004; Taylor et al., 2019), confinement in the protein pocket typically inhibits this pathway in favor of other energy dissipation routes. To rule out photoisomerization in the excited state and explore the photodynamics affected by halogenation, we implemented time-resolved electronic and vibrational spectroscopies (Liu et al., 2016; Fang et al., 2019). Using an fs photoexcitation pulse at $480 \mathrm{~nm}$ and a white light probe, TA spectra were collected to reveal dynamics in the first singlet excited state. The sfGFP, Cl-GFP, and Br-GFP all have a broad stimulated emission (SE) feature below $600 \mathrm{~nm}$ (in correlation with the fluorescence band in Figure 1, Figure S1) and a weak excited-state absorption band beyond $600 \mathrm{~nm}$ (Tang et al., 2015). We focus on TA dynamics by plotting the red-edge integrated signal of SE band from 550-570 nm, which rapidly reaches the maximum magnitude before a biexponential decay (see Figure 4D, Table S6). For sfGFP in $\mathrm{pH}=5.5$ buffer, only a small portion of the chromophore population is excited at $480 \mathrm{~nm}$, and a $\sim 1.2$ ps component accounts for $20 \%$ of the SE dynamics while a longer 1.2 nanosecond (ns) component is dominant. A similar time constant of 1.9 ps for the initial rise of fluorescence signal was reported in the deprotonated chromophore of GFP after $478 \mathrm{~nm}$ excitation (Chattoraj et al., 1996), likely arising from an ultrafast process (e.g., intramolecular vibrational relaxation; Felker and Zewail, 1985) that populates the fluorescent state of the deprotonated species. The long time component is less accurately determined due to the 600 ps detection window (see Section 2.2), but generally approaches the fluorescence lifetime $(\sim 3 \mathrm{~ns})$ of the photoexcited deprotonated chromophore (Chattoraj et al., 1996; Striker et al., 1999; Zimmer, 2002; Tang et al., 2018c).

Notably, the first recovery component of the SE band of halogenated proteins is significantly longer than the parent protein (see Figure 4D inset and Table S6), but the ns process is largely unchanged. The 4.1 ps component in Cl-GFP lengthens to $12.4 \mathrm{ps}$ in Br-GFP, and these time constants are attributable to the excited state $\left(S_{1}\right)$ relaxation dynamics 

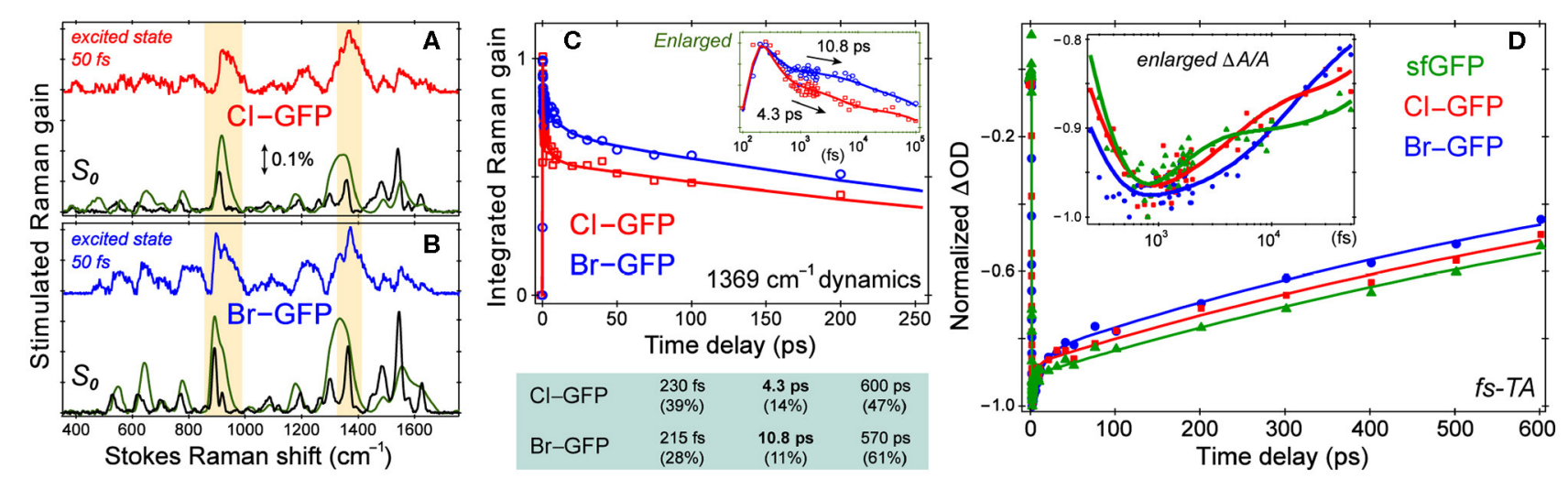

FIGURE 4 | Spectroscopic characterization of ncAA-GFPs. Ground and excited-state Stokes FSRS of (A) Cl-GFP and (B) Br-GFP. The spectra with the 555 and $507 \mathrm{~nm}$ Raman pump are depicted in black and green ( $\times 0.05)$, respectively. The excited-state Raman spectrum at $50 \mathrm{fs}$ after $480 \mathrm{~nm}$ photoexcitation is shown in red (Cl-GFP) and blue (Br-GFP). The stimulated Raman gain of $0.1 \%$ is indicated by the double-headed arrow. (C) Normalized Raman intensity dynamics of the 1,369 $\mathrm{cm}^{-1}$ band of Cl-GFP (red) and Br-GFP (blue) with the least-squares fit in solid lines. Early-time dynamics are highlighted in the inset on a semilogarithmic scale. The triexponential-fit components are listed below with the amplitude weight percentages of decay in parenthesis. (D) Fs-TA dynamics of the SE band (550-570 nm) of sfGFP (green), Cl-GFP (red), and Br-GFP (blue) in pH = 5.5 aqueous buffer solution following 480 nm excitation. The data are normalized at the maximal SE peak magnitude point for comparison. The least-squares fit for each data trace is shown as the color-coded solid curve. The inset shows the early-time dynamics on a semilogarithmic scale to highlight the multiple timescales involved.

other than fluorescence (vide infra), especially with the excess energy provided by the $480 \mathrm{~nm}$ pump. The excellent match between these TA time constants and the aforementioned Raman mode intensity decay time constants (Figure 4C, lower panel) supports a unified picture for energy relaxation on molecular timescales of a photoexcited deprotonated chromophore inside the protein pocket (Tang et al., 2018c; Fang et al., 2019). Previous photoelectron spectroscopic results of the UV-irradiated anionic HBDI chromophore (Mooney et al., 2013) and that with chemical modifications (e.g., difluoro-substituents) (Bochenkova et al., 2017) corroborate the changes in the excited-state energy surfaces and variation of the 1.4 ps lifetime (in gas phase and solution) following an initial $\sim 330$ fs component. Recently with timeresolved action spectroscopy, the HBDI anion after $480 \mathrm{~nm}$ pump shows ca. 1-11 ps lifetimes at $300 \mathrm{~K}$ (Svendsen et al., 2017).

To verify that the observed dynamics arise from a vibrational progression, we performed the time-resolved FSRS experiments in $S_{1}$ to directly track atomic motions (Fang et al., 2018). In previous FSRS reports on GFP derivatives, the modes with strong intensities are typically the phenol $\mathrm{C}-\mathrm{H}$ bending motions, phenolic $\mathrm{CO}$ stretch, and the imidazolinone $\mathrm{C}=\mathrm{N}$ stretch with frequencies at $\sim 1,180,1,265$, and $1,565 \mathrm{~cm}^{-1}$ in $S_{1}$ (Fang et al., 2009; Oscar et al., 2014; Tang et al., 2015, 2016). These high-frequency marker bands are also prominent in the preresonance ground-state Raman spectrum of sfGFP (Figure S4). Interestingly, halogenation changes this pattern by exhibiting several enhanced low-frequency modes. In the excited state, modes with major $\mathrm{C}-\mathrm{X}$ contributions exhibit the strongest intensities, and the mode frequencies are blue-shifted from those in $S_{0}$ (Figures 4A,B). Following $480 \mathrm{~nm}$ photoexcitation, the $S_{1}$ vibrational modes of $\mathrm{Cl}-\mathrm{GFP}$ and Br-GFP decay in time without the appearance of new peaks, while only small mode frequency blueshift occurs (Figure S6). The $\sim 1,369 \mathrm{~cm}^{-1}$ mode is present in $S_{0}$ and $S_{1}$ spectra of halogenated proteins and could be the vibronically coupled mode based on the energy gap observed in the electronic spectra (see Supplementary Text and Figure 1). This marker band dynamics are fit with a triexponential function (Figure 4C), largely matching the fs-TA dynamics (Figure 4D). Notably, Cl-GFP exhibits a $\sim 4$ ps decay time constant that is much shorter than the $\sim 11$ ps counterpart of Br-GFP, in correlation with the noticeable mode frequency blueshift in Figure S6 that indicates vibrational cooling in $\mathrm{S}_{1}$ (Fang et al., 2019).

Interestingly, the amplitude weights of the initial ps components (i.e., 20, 37, and 27\% in Table S6) correlate with the FQYs (i.e., 0.68, 0.88, and 0.76 in Table S2) of sfGFP, ClGFP, and Br-GFP, respectively. We surmise that the 4-12 ps components in Cl-GFP and Br-GFP (longer than 1.2 ps in sfGFP) involve certain nuclear motions associated with the phenolate ring as its size/weight increases, which allow effective vibrational cooling that promotes radiative emission from the lower portion of the potential energy surface of the deprotonated protein chromophore (Fang et al., 2018; Tang et al., 2018c). This mechanism is corroborated by a recent report on the introduction of asymmetric electronic structures and vibronic features to fluorophores, which can facilitate strong internal conversion with redder emission (Ren et al., 2018). Notably, photoisomerization typically leads to characteristic Raman mode frequency redshift due to the chromophore conformational change (Fang et al., 2019), which was not observed here (Figure S6). Moreover, the ring-twisting-induced non-radiative transition contradicts the high FQYs of halogenated sfGFP (Table S2), whereas the essentially non-fluorescent nitro-GFP likely involves an ultrafast nitroaromatic twisting motion leading to an $\mathrm{S}_{1} / \mathrm{S}_{0}$ conical intersection (Tang and Fang, 2019). One challenge that needs to be tackled before future FSRS experiments on nitro-GFP is the low signal-to-noise ratio without a prominent SE band (see Supplementary Text for details) like that in the 
halogenated sfGFP achieving resonance Raman enhancement in $\mathrm{S}_{1}$ (Figure S6).

\section{CONCLUSIONS}

In summary, we prepared and characterized a series of superfolder GFP mutants with ncAA chromophores using a combination of fs-TA spectroscopy, wavelength-tunable ground and excited-state FSRS (with ncAA chromophores in solution as control samples), and DFT calculations of normal mode frequencies. In particular, the single-site halogenated proteins display improved properties that include the redshifted absorption and emission, increased concentration of deprotonated emissive species, and an increased fluorescence quantum yield. Such desirable application properties of the halogenated GFP mutants stem from a solid biophysical chemistry foundation in that they are a direct consequence of the engineerable molecular structure and dynamics of the photosensitive unit inside a protein matrix. The nitroGFP provides a useful contrasting sample that will be further investigated.

We focused on the structural aspects of single-site halogenation at the protein active site to examine key conformational preference and elucidate the excited-state energy dissipation pathways in Cl-GFP and Br-GFP. Such a targeted analysis using a well-known series of electron-withdrawing groups with sufficient temporal and spectral resolution paints a more complete picture of chemically modified chromophores reacting to the incoming photons, thus enabling future rational design of functional molecular machines (Fang et al., 2019). The strong vibronic coupling that influences the SE dynamics may provide a useful direction to engineer probes for SE-depletion spectroscopy and imaging (Hell, 2009; Silva et al., 2016). Furthermore, these brighter protein mutants show that ncAA incorporation within the chromophore is a versatile and effective way to engineer photochemistry and protein functionality.

\section{REFERENCES}

Ai, H. W., Shaner, N. C., Cheng, Z., Tsien, R. Y., and Campbell, R. E. (2007). Exploration of new chromophore structures leads to the identification of improved blue fluorescent proteins. Biochemistry 46, 5904-5910. doi: 10.1021/bi700199g

Ayyadurai, N., Deepankumar, K., Prabhu, N. S., Budisa, N., and Yun, H. (2012). Evaluation and biosynthetic incorporation of chlorotyrosine into recombinant proteins. Biotechnol. Bioproc. Eng. 17, 679-686. doi: 10.1007/s12257-012-0066-6

Ayyadurai, N., Saravanan Prabhu, N., Deepankumar, K., Lee, S.-G., Jeong, H.H., Lee, C.-S., et al. (2011). Development of a selective, sensitive, and reversible biosensor by the genetic incorporation of a metal-binding site into green fluorescent protein. Angew. Chem. Int. Ed. 50, 6534-6537. doi: 10.1002/anie.201008289

Bae, J. H., Paramita Pal, P., Moroder, L., Huber, R., and Budisa, N. (2004). Crystallographic evidence for isomeric chromophores in 3-fluorotyrosyl-green fluorescent protein. ChemBioChem 5, 720-722. doi: 10.1002/cbic.200300818

Baird, G. S., Zacharias, D. A., and Tsien, R. Y. (1999). Circular permutation and receptor insertion within green fluorescent proteins. Proc. Natl. Acad. Sci. U. S. A. 96, 11241-11246. doi: 10.1073/pnas.96.20.11241

\section{DATA AVAILABILITY STATEMENT}

The raw data supporting the conclusions of this article will be made available by the authors, without undue reservation.

\section{AUTHOR CONTRIBUTIONS}

$\mathrm{RM}$ and $\mathrm{CF}$ conceived and designed the research and acquired funding. $\mathrm{BO}$ and $\mathrm{LZ}$ performed spectroscopic experiments. $\mathrm{BO}, \mathrm{NR}, \mathrm{AC}$, and JS performed data curation including calculations. HW, JP, and KS contributed new protein and chromophore samples. LZ and CF contributed advanced nonlinear spectroscopic tools. $\mathrm{BO}$ and $\mathrm{CF}$ wrote the manuscript. All the authors have edited the final manuscript and approved it for publication.

\section{FUNDING}

CF was supported by the US National Science Foundation through a Career Grant (CHE-1455353). We thank the Betty Wang Discovery Fund (2019 to CF) at the OSU College of Science for the acquisition of a new Shimadzu RF-6000 spectrofluorophotometer. RM was supported by a National Institutes of Health Grant (RGM114653A) and National Science Foundation Grant (MCB-1518265).

\section{ACKNOWLEDGMENTS}

We thank Dr. Longteng Tang, Cheng Chen, Taylor Krueger, Sean Tachibana, and Sean Boulanger for helpful discussions.

\section{SUPPLEMENTARY MATERIAL}

The Supplementary Material for this article can be found online at: https://www.frontiersin.org/articles/10.3389/fmolb. 2020.00131/full\#supplementary-material

Baldridge, A., Kowalik, J., and Tolbert, L. M. (2010). Efficient synthesis of new 4-arylideneimidazolin-5-ones related to the GFP chromophore by $2+3$ cyclocondensation of arylideneimines with imidate ylides. Synthesis 2010, 2424-2436. doi: 10.1055/s-0029-1218796

Bell, A. F., He, X., Wachter, R. M., and Tonge, P. J. (2000). Probing the ground state structure of the green fluorescent protein chromophore using Raman spectroscopy. Biochemistry 39, 4423-4431. doi: 10.1021/bi992675o

Betzig, E., Patterson, G. H., Sougrat, R., Lindwasser, O. W., Olenych, S., Bonifacino, J. S., et al. (2006). Imaging intracellular fluorescent proteins at nanometer resolution. Science 313, 1642-1645. doi: 10.1126/science.1127344

Bochenkova, A. V., Mooney, C. R. S., Parkes, M. A., Woodhouse, J. L., Zhang, L., Lewin, R., et al. (2017). Mechanism of resonant electron emission from the deprotonated GFP chromophore and its biomimetics. Chem. Sci. 8, 3154-3163. doi: 10.1039/C6SC05529J

Brejc, K., Sixma, T. K., Kitts, P. A., Kain, S. R., Tsien, R. Y., Orm,ö, M., et al. (1997). Structural basis for dual excitation and photoisomerization of the Aequorea victoria green fluorescent protein. Proc. Natl. Acad. Sci. U. S. A. 94, 2306-2311. doi: 10.1073/pnas.94.6.2306

Chalfie, M., Tu, Y., Euskirchen, G., Ward, W. W., and Prasher, D. C. (1994). Green fluorescent protein as a marker for gene expression. Science 263, 802-805. doi: 10.1126/science.8303295 
Chang, J., Romei, M. G., and Boxer, S. G. (2019). Structural evidence of photoisomerization pathways in fluorescent proteins. J. Am. Chem. Soc. 141, 15504-15508. doi: 10.1021/jacs.9b08356

Chattoraj, M., King, B. A., Bublitz, G. U., and Boxer, S. G. (1996). Ultra-fast excited state dynamics in green fluorescent protein: multiple states and proton transfer. Proc. Natl. Acad. Sci. U. S. A. 93, 8362-8367. doi: 10.1073/pnas.93.16.8362

Chen, C., Zhu, L., Baranov, M. S., Tang, L., Baleeva, N. S., Smirnov, A. Y., et al. (2019). Photoinduced proton transfer of GFP-inspired fluorescent superphotoacids: principles and design. J. Phys. Chem. B 123, 3804-3821. doi: 10.1021/acs.jpcb.9b03201

Chen, C., Zhu, L., Boulanger, S. A., Baleeva, N. S., Myasnyanko, I. N., Baranov, M. S., et al. (2020). Ultrafast excited-state proton transfer dynamics in dihalogenated non-fluorescent and fluorescent GFP chromophores. J. Chem. Phys. 152:021101. doi: 10.1063/1.5138666

Chen, C., Zhu, L., and Fang, C. (2018). Femtosecond stimulated Raman line shapes: dependence on resonance conditions of pump and probe pulses. Chin. J. Chem. Phys. 31, 492-502. doi: 10.1063/1674-0068/31/cjcp1805125

Cooley, R. B., Feldman, J. L., Driggers, C. M., Bundy, T. A., Stokes, A. L., Karplus, P. A., et al. (2014). Structural basis of improved secondgeneration 3-nitro-tyrosine tRNA synthetases. Biochemistry 53, 1916-1924. doi: 10.1021/bi5001239

De Filippis, V., Frasson, R., and Fontana, A. (2006). 3-Nitrotyrosine as a spectroscopic probe for investigating protein-protein interactions. Protein Sci. 15, 976-986. doi: 10.1110/ps.051957006

Dedecker, P., De Schryver, F. C., and Hofkens, J. (2013). Fluorescent proteins: shine on, you crazy diamond. J. Am. Chem. Soc. 135, 2387-2402. doi: $10.1021 /$ ja309768d

Dietze, D. R., and Mathies, R. A. (2016). Femtosecond stimulated Raman spectroscopy. ChemPhysChem 17, 1224-1251. doi: 10.1002/cphc.2016 00104

Duus, J. Ø., Meldal, M., and Winkler, J. R. (1998). Fluorescence energy-transfer probes of conformation in peptides: the 2-aminobenzamide/nitrotyrosine pair. J. Phys. Chem. B 102, 6413-6418. doi: 10.1021/jp973352+

Fang, C., Frontiera, R. R., Tran, R., and Mathies, R. A. (2009). Mapping GFP structure evolution during proton transfer with femtosecond Raman spectroscopy. Nature 462, 200-204. doi: 10.1038/nature08527

Fang, C., Tang, L., and Chen, C. (2019). Unveiling coupled electronic and vibrational motions of chromophores in condensed phases. J. Chem. Phys. 151:200901. doi: 10.1063/1.5128388

Fang, C., Tang, L., Oscar, B. G., and Chen, C. (2018). Capturing structural snapshots during photochemical reactions with ultrafast Raman spectroscopy: from materials transformation to biosensor responses. J. Phys. Chem. Lett. 9, 3253-3263. doi: 10.1021/acs.jpclett.8b00373

Felker, P. M., and Zewail, A. H. (1985). Dynamics of intramolecular vibrationalenergy redistribution (IVR). I. Coherence effects. J. Chem. Phys. 82, 2961-2974. doi: $10.1063 / 1.448246$

Fleissner, M. R., Brustad, E. M., Kálai, T., Altenbach, C., Cascio, D., Peters, F. B., et al. (2009). Site-directed spin labeling of a genetically encoded unnatural amino acid. Proc. Natl. Acad. Sci. U. S. A. 106, 21637-21642. doi: 10.1073/pnas.0912009106

Gross, L. A., Baird, G. S., Hoffman, R. C., Baldridge, K. K., and Tsien, R. Y. (2000). The structure of the chromophore within DsRed, a red fluorescent protein from coral. Proc. Natl. Acad. Sci. U. S. A. 97, 11990-11995. doi: 10.1073/pnas.97.22.11990

Hall, C. R., Tolentino Collado, J., Iuliano, J. N., Gil, A. A., Adamczyk, K., Lukacs, A., et al. (2019). Site-specific protein dynamics probed by ultrafast infrared spectroscopy of a non-canonical amino acid. J. Phys. Chem. B 123, 9592-9597. doi: 10.1021/acs.jpcb.9b09425

Hasegawa, J. Y., Fujimoto, K., Swerts, B., Miyahara, T., and Nakatsuji, H. (2007). Excited states of GFP chromophore and active site studied by the SAC-CI method: effect of protein-environment and mutations. J. Comput. Chem. 28, 2443-2452. doi: 10.1002/jcc.20667

Hell, S. W. (2009). Microscopy and its focal switch. Nat. Meth. 6, 24-32. doi: 10.1038/nmeth.1291

Hyun Bae, J., Rubini, M., Jung, G., Wiegand, G., Seifert, M. H. J., Azim, M. K., et al. (2003). Expansion of the genetic code enables design of a novel "gold" class of green fluorescent proteins. J. Mol. Biol. 328, 1071-1081. doi: 10.1016/S0022-2836(03)00364-4
Jang, H. S., Gu, X., Cooley, R. B., Porter, J. J., Henson, R. L., Willi, T., et al. (2020). Efficient site-specific prokaryotic and eukaryotic incorporation of halotyrosine amino acids into proteins. ACS Chem. Biol. 15, 562-574. doi: 10.1021/acschembio.9b01026

Jung, G. (2012a). Fluorescent Proteins I: From Understanding to Design. (Berlin, Heidelberg: Springer-Verlag).

Jung, G. (2012b). Fluorescent Proteins II: Application of Fluorescent Protein Technology. (Berlin, Heidelberg: Springer-Verlag).

Kovács, A., Izvekov, V., Keresztury, G., and Pongor, G. (1998). Vibrational analysis of 2-nitrophenol. A joint FT-IR, FT-Raman and scaled quantum mechanical study. Chem. Phys. 238, 231-243. doi: 10.1016/S0301-0104(98)00307-3

Kummer, A. D., Wiehler, J., Schüttrigkeit, T. A., Berger, B. W., Steipe, B., and Michel-Beyerle, M. E. (2002). Picosecond time-resolved fluorescence from blue-emitting chromophore variants $\mathrm{Y} 66 \mathrm{~F}$ and $\mathrm{Y} 66 \mathrm{H}$ of the green fluorescent protein. ChemBioChem 3, 659-663. doi: 10.1002/14397633(20020703)3:7<659::AID-CBIC659>3.0.CO;2-U Available online at: https://chemistry-europe.onlinelibrary.wiley.com/doi/abs/10.1002/1439-7633 \%2820020703\%293\%3A7\%3C659\%3A\%3AAID-CBIC659\%3E3.0.CO\%3B2-U

Link, A. J., Mock, M. L., and Tirrell, D. A. (2003). Non-canonical amino acids in protein engineering. Curr. Opin. Biotechnol. 14, 603-609. doi: 10.1016/j.copbio.2003.10.011

Liu, W., Wang, Y., Tang, L., Oscar, B. G., Zhu, L., and Fang, C. (2016). Panoramic portrait of primary molecular events preceding excited state proton transfer in water. Chem. Sci. 7, 5484-5494. doi: 10.1039/C6SC00672H

Lossau, H., Kummer, A., Heinecke, R., Pollinger-Dammer, F., Kompa, C., Bieser, G., et al. (1996). Time-resolved spectroscopy of wild-type and mutant green fluorescent proteins reveals excited state deprotonation consistent with fluorophore-protein interactions. Chem. Phys. 213, 1-16. doi: 10.1016/S0301-0104(96)00340-0

Malde, A. K., Zuo, L., Breeze, M., Stroet, M., Poger, D., Nair, P. C., et al. (2011). An automated force field topology builder (ATB) and repository: version 1.0. J. Chem. Theory Comput. 7, 4026-4037. doi: 10.1021/ct200196m

Mandal, D., Tahara, T., and Meech, S. R. (2004). Excited-state dynamics in the green fluorescent protein chromophore. J. Phys. Chem. B 108, 1102-1108. doi: $10.1021 /$ jp035816b

Meneely, C. T., She, C. Y., and Edwards, D. F. (1971). Line-shape parameters of the Raman lines of chloro-, bromo-, and iodobenzene. J. Mol. Spectrosc. 39, 73-78. doi: 10.1016/0022-2852(71)90277-3

Merrick, J. P., Moran, D., and Radom, L. (2007). An evaluation of harmonic vibrational frequency scale factors. J. Phys. Chem. A 111, 11683-11700. doi: 10.1021/jp073974n

Mooney, C. R. S., Horke, D. A., Chatterley, A. S., Simperler, A., Fielding, H. H., and Verlet, J. R. R. (2013). Taking the green fluorescence out of the protein: dynamics of the isolated GFP chromophore anion. Chem. Sci. 4, 921-927. doi: $10.1039 / \mathrm{C} 2 \mathrm{SC} 21737 \mathrm{~F}$

Oltrogge, L. M., and Boxer, S. G. (2015). Short hydrogen bonds and proton delocalization in green fluorescent protein (GFP). ACS Cent. Sci. 1, 148-156. doi: 10.1021/acscentsci.5b00160

Ormö, M., Cubitt, A. B., Kallio, K., Gross, L. A., Tsien, R. Y., and Remington, S. J. (1996). Crystal structure of the Aequorea victoria green fluorescent protein. Science 273, 1392-1395. doi: 10.1126/science.273.5280.1392

Oscar, B. G., Liu, W., Zhao, Y., Tang, L., Wang, Y., Campbell, R. E., et al. (2014). Excited-state structural dynamics of a dual-emission calmodulin-green fluorescent protein sensor for calcium ion imaging. Proc. Natl. Acad. Sci. U. S. A. 111, 10191-10196. doi: 10.1073/pnas.1403712111

Pal, P. P., Bae, J. H., Azim, M. K., Hess, P., Friedrich, R., Huber, R., et al. (2005). Structural and spectral response of Aequorea victoria green fluorescent proteins to chromophore fluorination. Biochemistry 44, 3663-3672. doi: 10.1021/bi0484825

Patterson, G. H., Knobel, S. M., Sharif, W. D., Kain, S. R., and Piston, D. W. (1997) Use of the green fluorescent protein and its mutants in quantitative fluorescence microscopy. Biophys. J. 73, 2782-2790. doi: 10.1016/S0006-3495(97)78307-3

Patterson, G. H., and Lippincott-Schwartz, J. (2002). A photoactivatable GFP for selective photolabeling of proteins and cells. Science 297, 1873-1877. doi: $10.1126 /$ science. 1074952

Pédelacq, J.-D., Cabantous, S., Tran, T., Terwilliger, T. C., and Waldo, G. S. (2006). Engineering and characterization of a superfolder green fluorescent protein. Nat. Biotechnol. 24, 79-88. doi: 10.1038/nbt1172 
Peeler, J. C., and Mehl, R. A. (2012). "Site-specific incorporation of unnatural amino acids as probes for protein conformational changes," in Unnatural Amino Acids: Methods and Protocols, eds L. Pollegioni, and S. Servi (New York, NY: Humana Press), 125-134.

Piatkevich, K. D., Malashkevich, V. N., Almo, S. C., and Verkhusha, V. V. (2010). Engineering ESPT pathways based on structural analysis of LSSmKate red fluorescent proteins with large Stokes shift. J. Am. Chem. Soc. 132, 10762-10770. doi: 10.1021/ja101974k

Quick, M., Dobryakov, A. L., Kovalenko, S. A., and Ernsting, N. P. (2015). Resonance femtosecond-stimulated Raman spectroscopy without actinic excitation showing low-frequency vibrational activity in the $\mathrm{S}_{2}$ state of all-trans $\beta$-carotene. J. Phys. Chem. Lett. 6, 1216-1220. doi: 10.1021/acs.jpclett.5b00243

Rauch, B. J., Porter, J. J., Mehl, R. A., and Perona, J. J. (2016). Improved incorporation of non-canonical amino acids by an engineered tRNATyr suppressor. Biochemistry 55, 618-628. doi: 10.1021/acs.biochem.5b01185

Reddington, S. C., Rizkallah, P. J., Watson, P. D., Pearson, R., Tippmann, E. M., and Jones, D. D. (2013). Different photochemical events of a genetically encoded phenyl azide define and modulate GFP fluorescence. Angew. Chem. Int. Ed. 52, 5974-5977. doi: 10.1002/anie.201301490

Ren, T.-B., Xu, W., Zhang, W., Zhang, X.-X., Wang, Z.-Y., Xiang, Z., et al. (2018). A general method to increase Stokes shift by introducing alternating vibronic structures. J. Am. Chem. Soc. 140, 7716-7722. doi: 10.1021/jacs.8b04404

Schellenberg, P., Johnson, E., Esposito, A. P., Reid, P. J., and Parson, W. W. (2001). Resonance Raman scattering by the green fluorescent protein and an analogue of its chromophore. J. Phys. Chem. B 105, 5316-5322. doi: 10.1021/jp0046243

Seeliger, D., and de Groot, B. L. (2010). Protein thermostability calculations using alchemical free energy simulations. Biophys. J. 98, 2309-2316. doi: 10.1016/j.bpj.2010.01.051

Shaner, N. C., Campbell, R. E., Steinbach, P. A., Giepmans, B. N. G., Palmer, A. E., and Tsien, R. Y. (2004). Improved monomeric red, orange and yellow fluorescent proteins derived from Discosoma sp. red fluorescent protein. Nat. Biotechnol. 22, 1567-1572. doi: 10.1038/nbt1037

Shimomura, O., Johnson, F. H., and Saiga, Y. (1962). Extraction, purification and properties of Aequorin, a bioluminescent protein from the luminous Hydromedusan, Aequorea. J. Cell. Comp. Physiol. 59, 223-239. doi: 10.1002/jcp.1030590302

Silva, W. R., Graefe, C. T., and Frontiera, R. R. (2016). Toward label-free super-resolution microscopy. ACS Photonics 3, 79-86. doi: 10.1021/acsphotonics.5b00467

Sripakdeevong, P., Cevec, M., Chang, A. T., Erat, M. C., Ziegeler, M., Zhao, Q., et al. (2014). Structure determination of non-canonical RNA motifs guided by ${ }^{1}$ H NMR chemical shifts. Nat. Methods 11, 413-416. doi: 10.1038/nmeth.2876

Striker, G., Subramaniam, V., Seidel, C. A. M., and Volkmer, A. (1999). Photochromicity and fluorescence lifetimes of green fluorescent protein. J. Phys. Chem. B 103, 8612-8617. doi: 10.1021/jp991425e

Subach, F. V., and Verkhusha, V. V. (2012). Chromophore transformations in red fluorescent proteins. Chem. Rev. 112, 4308-4327. doi: 10.1021/cr2001965

Svendsen, A., Kiefer, H. V., Pedersen, H. B., Bochenkova, A. V., and Andersen, L. H. (2017). Origin of the intrinsic fluorescence of the green fluorescent protein. J. Am. Chem. Soc. 139, 8766-8771. doi: 10.1021/jacs.7b04987

Tang, L., and Fang, C. (2019). Nitration of tyrosine channels photoenergy through a conical intersection in water. J. Phys. Chem. B 123, 4915-4928. doi: 10.1021/acs.jpcb.9b03464

Tang, L., Liu, W., Wang, Y., Zhao, Y., Oscar, B. G., Campbell, R. E., et al. (2015). Unraveling ultrafast photoinduced proton transfer dynamics in a fluorescent protein biosensor for $\mathrm{Ca}^{2+}$ imaging. Chem. Eur. J. 21, 6481-6490. doi: 10.1002/chem.201500491

Tang, L., Liu, W., Wang, Y., Zhu, L., Han, F., and Fang, C. (2016). Ultrafast structural evolution and chromophore inhomogeneity inside a greenfluorescent-protein-based $\mathrm{Ca}^{2+}$ biosensor. J. Phys. Chem. Lett. 7, 1225-1230. doi: 10.1021/acs.jpclett.6b00236

Tang, L., Wang, Y., Zhu, L., Kallio, K., Remington, S. J., and Fang, C. (2018a). Photoinduced proton transfer inside an engineered green fluorescent protein: a stepwise-concerted-hybrid reaction. Phys. Chem. Chem. Phys. 20, 12517-12526. doi: 10.1039/C8CP01907J

Tang, L., Zhu, L., Taylor, M. A., Wang, Y., Remington, S. J., and Fang, C. (2018b). Excited state structural evolution of a GFP single-site mutant tracked by tunable femtosecond-stimulated Raman spectroscopy. Molecules 23:2226 doi: 10.3390/molecules 23092226

Tang, L., Zhu, L., Wang, Y., and Fang, C. (2018c). Uncovering the hidden excited state toward fluorescence of an intracellular $\mathrm{pH}$ indicator. J. Phys. Chem. Lett. 9, 4969-4975. doi: 10.1021/acs.jpclett.8b02281

Taylor, M. A., Zhu, L., Rozanov, N. D., Stout, K. T., Chen, C., and Fang, C. (2019). Delayed vibrational modulation of the solvated GFP chromophore into a conical intersection. Phys. Chem. Chem. Phys. 21, 9728-9739. doi: 10.1039/C9CP01077G

Tomosugi, W., Matsuda, T., Tani, T., Nemoto, T., Kotera, I., Saito, K., et al. (2009). An ultramarine fluorescent protein with increased photostability and $\mathrm{pH}$ insensitivity. Nat. Methods 6, 351-353. doi: 10.1038/nmet h. 1317

Tozzini, V., and Nifosì, R. (2001). Ab initio molecular dynamics of the green fluorescent protein (GFP) chromophore: an insight into the photoinduced dynamics of green fluorescent proteins. J. Phys. Chem. B 105, 5797-5803. doi: $10.1021 /$ jp010052q

Tsien, R. Y. (1998). The green fluorescent protein. Annu. Rev. Biochem. 67, 509-544. doi: 10.1146/annurev.biochem.67.1.509

Vengris, M., van Stokkum, I. H. M., He, X., Bell, A. F., Tonge, P. J., van Grondelle, R., et al. (2004). Ultrafast excited and ground-state dynamics of the green fluorescent protein chromophore in solution. J. Phys. Chem. A 108, 4587-4598. doi: 10.1021/jp037902h

Wachter, R. M., Elsliger, M.-A., Kallio, K., Hanson, G. T., and Remington, S. J. (1998). Structural basis of spectral shifts in the yellow-emission variants of green fluorescent protein. Structure 6, 1267-1277. doi: 10.1016/S0969-2126(98)00127-0

Wachter, R. M., King, B. A., Heim, R., Kallio, K., Tsien, R. Y., Boxer, S. G., et al. (1997). Crystal structure and photodynamic behavior of the blue emission variant Y66H/Y145F of green fluorescent protein. Biochemistry 36, 9759-9765. doi: 10.1021/bi970563w

Wang, L., Xie, J. M., Deniz, A. A., and Schultz, P. G. (2003). Unnatural amino acid mutagenesis of green fluorescent protein. J. Org. Chem. 68, 174-176. doi: $10.1021 /$ jo026570u

Wang, Y., Tang, L., Liu, W., Zhao, Y., Oscar, B. G., Campbell, R. E., et al. (2015). Excited state structural events of a dual-emission fluorescent protein biosensor for $\mathrm{Ca}^{2+}$ imaging studied by femtosecond stimulated Raman spectroscopy. J. Phys. Chem. B 119, 2204-2218. doi: 10.1021/jp50 $5698 \mathrm{z}$

Young, D. D., Jockush, S., Turro, N. J., and Schultz, P. G. (2011). Synthetase polyspecificity as a tool to modulate protein function. Bioorg. Med. Chem. Lett. 21, 7502-7504. doi: 10.1016/j.bmcl.2011. 09.108

Zhang, W., Wang, Y. Q., and Wang, J. Y. (2012). Fluorescent protein engineering through genetic incorporation of 3-chlorotyrosine. Prog. Biochem. Biophys. 39, 378-387. doi: 10.3724/SP.J.1206.2011.00299

Zhao, Y., Araki, S., Wu, J., Teramoto, T., Chang, Y.-F., Nakano, M., et al. (2011). An expanded palette of genetically encoded $\mathrm{Ca}^{2+}$ indicators. Science 333, 1888-1891. doi: 10.1126/science.1208592

Zhu, L., Liu, W., and Fang, C. (2014). A versatile femtosecond stimulated Raman spectroscopy setup with tunable pulses in the visible to near infrared. Appl. Phys. Lett. 105, 041106. doi: 10.1063/1.4891766

Zimmer, M. (2002). Green fluorescent protein (GFP): applications, structure, and related photophysical behavior. Chem. Rev. 102, 759-782. doi: $10.1021 / \mathrm{cr} 010142 \mathrm{r}$

Conflict of Interest: The authors declare that the research was conducted in the absence of any commercial or financial relationships that could be construed as a potential conflict of interest.

Copyright (C) 2020 Oscar, Zhu, Wolfendeen, Rozanov, Chang, Stout, Sandwisch, Porter, Mehl and Fang. This is an open-access article distributed under the terms of the Creative Commons Attribution License (CC BY). The use, distribution or reproduction in other forums is permitted, provided the original author(s) and the copyright owner(s) are credited and that the original publication in this journal is cited, in accordance with accepted academic practice. No use, distribution or reproduction is permitted which does not comply with these terms. 